\title{
Relationship between the Expression of Advanced Glycation End-Products (AGE) and the Receptor for AGE (RAGE) mRNA in Diabetic Nephropathy
}

\author{
Daisuke Suzuki, Masao Toyoda, Naoyuki Yamamoto, Masaaki Miyauchi, Mayuko Katoh, \\ Moritsugu Kimura, Mayumi Maruyama, Masashi Honma, \\ Tomoya Umezono and Mitsunori Yagame
}

\begin{abstract}
Objective The receptor for advanced glycation end-products (RAGE) is one of several advanced glycation end-product (AGE)-specific cellular receptors. To evaluate the relationship between AGE and RAGE in renal tissues of diabetic nephropathy (DN), we examined the levels of expression of AGE protein and of RAGE mRNA. We also investigated the relationships among the degree of mesangial expansion and the expression of AGE and RAGE mRNA.

Patients and Methods Renal biopsy tissues were obtained from 20 patients with DN. We performed immunohistochemical staining using monoclonal anti-AGE antibody and in situ hybridization using non-radioactive oligonucleotide RAGE probe on these tissues. We also examined five control renal samples. We evaluated the intensity of positive anti-AGE antibody staining and the percentage of cells positive for RAGE mRNA. We also measured the total glomerular area and mesangial area in glomeruli using an automatic image analyzer. We then calculated the percentage of mesangial area as a proportion of the total glomerular area (\%Mes).

Results Anti-AGE antibody was detected in the expanded mesangial matrix in DN but not in control samples. RAGE mRNA expression was detected mainly in glomerular intrinsic cells, including glomerular mesangial and epithelial cells, in both DN and control. \% Mes correlated significantly with both the intensity of anti-AGE antibody positive staining and the percentage of cells positive for RAGE mRNA.

Conclusions Our findings suggest that both AGE and RAGE are associated with the development and progression of DN.
\end{abstract}

Key words: Advanced glycation end-products (AGE), receptor for advanced glycation end-products (RAGE), diabetic nephropathy, in situ hybridization

(DOI: 10.2169/internalmedicine.45.1557)

\section{Introduction}

Diabetic nephropathy (DN) is one of the major complications of both types 1 and 2 diabetes mellitus and the most common cause of end-stage renal failure in many countries. The pathologic changes of DN include progressive expansion of the mesangial matrix and thickening of the glomerular basement membrane (GBM) (1-3). Mesangial expansion is thought to be the most important lesion, and was reported to be the critical lesion that ultimately leads to renal insufficiency in DN (1-3).

Various pathogenic mechanisms have been postulated for DN, although the precise mechanism remains to be clarified. Long-term metabolic aberrations are considered to play a key role, because a number of changes in the kidney are prevented or improved after normalization of glucose metabolism in animal and human models $(4,5)$. Possible me-

Division of Nephrology and Metabolism, Department of Internal Medicine, School of Medicine, Tokai University, Isehara Received for publication August 30, 2005; Accepted for publication January 18, 2006 Correspondence to Daisuke Suzuki, Division of Nephrology and Metabolism, Department of Internal Medicine, School of Medicine, Tokai University, Bohseidai, Isehara, Kanagawa 259-1193 
Table 1. Clinical Parameters of Diabetic Patients and Normal Subjects

\begin{tabular}{lll} 
& DN & Controls \\
\hline Gender (M/F) & $16 / 4$ & $4 / 1$ \\
Age (yr) & $42.5 \pm 11.8$ & $60.0 \pm 8.1^{*}$ \\
Known duration (yr) & $9.8 \pm 5.7$ & \\
SBP (mmHg) & $141.0 \pm 17.1$ & $128.8 \pm 14.1$ \\
DBP (mmHg) & $83.4 \pm 10.8$ & $71.2 \pm 14.7$ \\
S-Cr (mg/dl) & $0.81 \pm 0.16$ & $0.80 \pm 0.16$ \\
TP (g/dl) & $6.32 \pm 1.00$ & $6.54 \pm 0.27$ \\
HbAlc (\%) & $9.65 \pm 2.58$ & not done \\
U-protein (g/d) & $0.73 \pm 1.02^{*}$ & $0.02 \pm 0.01$ \\
Ccr (ml/min) & $87.6 \pm 30.8$ & not done \\
\hline
\end{tabular}

DN; diabetic nephropathy. SBP; systolic blood pressure, DBP; diastolic blood pressure, TP; total protein, Ccr; creatinine clearance, "; p<0.01

diators of untoward effects of hyperglycemia include advanced glycation end-products (AGE) generated by the Maillard reaction through non-enzymatic glycation of protein amino groups (6). Recently, we reported that AGE accumulate in the tissues of $\mathrm{DN}(7,8)$ and that their accumulation correlates with the severity of diabetic complications $(9$, 10). Several lines of evidence suggest that AGE may be involved in the development of diabetic glomerular lesions (7, $8,11,12)$. AGE modification indeed alters the structure and function of matrix tissue proteins (6) and, more interestingly, AGE-modified proteins stimulate a variety of cellular responses via a specific cell-surface receptor $(13,14)$ on several cell types, including glomerular cells (15-17). The receptor for AGE (RAGE) is one of several AGE-specific cellular receptors. RAGE was first identified as a receptor for AGE using bovine lung extract. It is a signal transduction receptor for AGE of the immunoglobulin super-family that mediates diverse cellular responses $(13,14)$. Recently, RAGE has been proposed to play an important role in the development of DN $(18,19)$. However, the AGE-RAGE interaction is not yet fully understood.

The aim of the present study was to evaluate the relationship between AGE and RAGE in tissues from DN. We examined AGE expression using immunohistochemical staining and RAGE mRNA expression using an in situ hybridization technique. We also investigated the relationships among the degree of mesangial expansion and the expression of AGE and RAGE mRNA.

\section{Methods}

\section{Patients}

Renal biopsy specimens were obtained from 20 patients with $\mathrm{DN}(3,20)$. All diabetic patients had macro proteinuria. The presence of DN was confirmed by pathological evalu- ation of renal biopsy specimens, using techniques such as light microscopy, electron microscopy and immunofluorescence staining. The following clinical parameters were recorded at the time of renal biopsy: gender, age, known duration, blood pressure, serum creatinine, total protein, hemoglobin A1c, urinary protein and creatinine clearance. Clinical features are shown in Table 1. The study was approved by the Human Research Committee of Tokai University School of Medicine, and informed consent was obtained from each patient for renal biopsy and immunohistochemical staining and in situ hybridization studies. We also examined five control samples obtained from five subjects using unaffected portions of surgically removed kidneys afflicted with malignancies. After resection, the samples were embedded in OCT compound (Tissue Tek, Miles, Elkhart, IN) and stored until use. The control patients had no urinary abnormalities, and histopathological examination of control tissues excluded any glomerular disease.

\section{Measurement of the percentage of mesangial area with respect to total glomerular area}

We calculated the percentage of mesangial area with respect to total glomerular area to determine the degree of mesangial expansion in renal tissues. To minimize bias, 10 glomeruli were chosen from more than 50 glomeruli in each patient and in control samples. The criterion for selection was the cross-section through their vascular poles. We measured the total glomerular area and periodic acid-Schiff (PAS)-positive area using an automatic image analyzer determined by three independent investigators. We then calculated the percentage of mesangial area with respect to total glomerular area (\%Mes).

\section{Immunohistochemical staining}

Renal biopsy specimens were sectioned at $4 \mu \mathrm{m}$ and fixed with $4 \%$ paraformaldehyde in phosphate-buffered saline (PBS) on ice for 15 minutes. After washing with PBS, the sections were blocked with $4 \%$ skim milk for 60 minutes at room temperature and incubated with peroxidase (HRP)conjugated anti-AGE antibody (Transgenic, Kumamoto, Japan) at $4{ }^{\circ} \mathrm{C}$ overnight. The major epitope-structure of this antibody was recently identified as $\mathrm{N}^{\varepsilon}$-carboxymethyllysine (CML) (21). After washing with Tris-saline buffer containing $100 \mathrm{mM} \mathrm{NaCl}$ and $150 \mathrm{mM}$ Tris- $\mathrm{HCl}, \mathrm{pH} 7.5$, the sections were dehydrated through a graded ethanol series, incubated in methanol with $0.3 \% \mathrm{H}_{2} \mathrm{O}_{2}$ at room temperature for 20 minutes to block endogenous peroxidase, and washed three times with Tris-saline buffer with $0.02 \%$ Tween 20 (Wako Pure Chemical Industries, Osaka, Japan). After a final wash with Tris-saline buffer containing Tween 20, they were developed by reaction with diaminobenzidine tetrahydrochloride in $0.05 \mathrm{M}$ Tris- $\mathrm{HCl}, \mathrm{pH} \mathrm{7.6}$, and $0.03 \% \mathrm{H}_{2} \mathrm{O}_{2}$. Sections were counterstained briefly with hematoxylin, and then rinsed, dehydrated, cleared in xylene and mounted. The intensity of staining for anti-AGE antibody was evaluated using a score of 0-4 (negative staining: 0, trace: 1 , weak: 2 , 

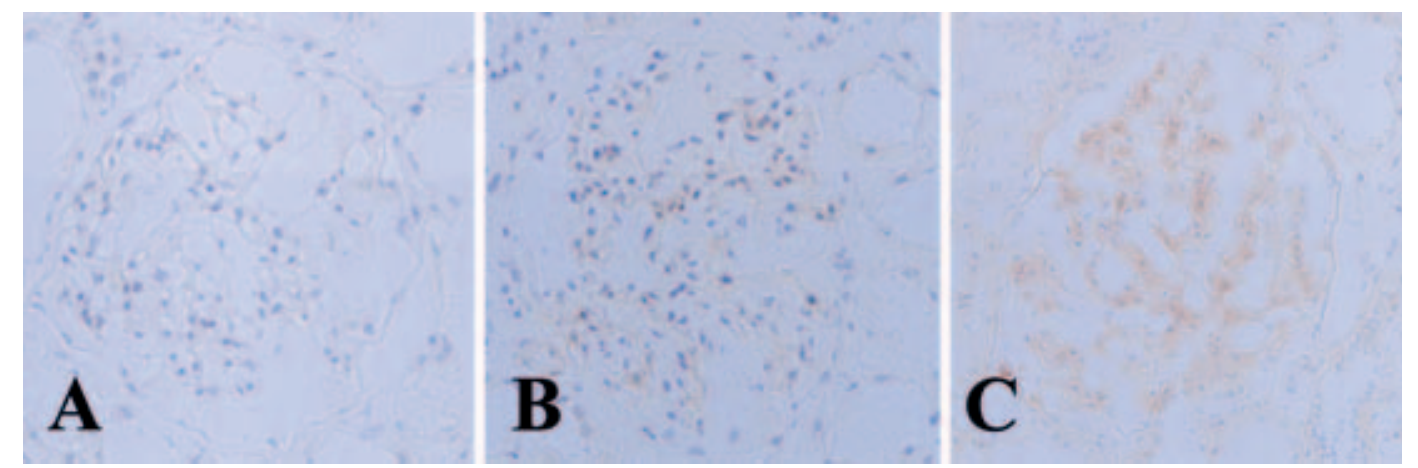

Figure 1. Immunohistochemical detection of anti-AGE antibody in controls (A) and diabetic nephropathy ( $B$, with mild mesangial expansion and $C$, with moderate mesangial expansion). The AGE antibodies did not stain the glomeruli in control subjects (A). In contrast, in the glomeruli of diabetic nephropathy, AGE stained positively in the expanded mesangial area (B and C). (Original magnification $\times 100$ )

Table 2. Results of \% Mes, Int AGE ab and \% RAGE mRNA in DN and Controls

\begin{tabular}{lcc} 
& DN & Controls \\
\hline$\%$ Mes & $23.5 \pm 4.8^{*}$ & $14.3 \pm 1.6$ \\
Int AGE ab & $3.1 \pm 0.7^{*}$ & $0.0 \pm 0.0$ \\
\% RAGE mRNA & $29.6 \pm 5.2^{*}$ & $12.0 \pm 2.1$ \\
\hline
\end{tabular}

DN; diabetic nephropathy

\% Mes: the percentage of mesangial area with respeet to the total glomerular area Int $\mathrm{AGE}$ ab: the intensity of anti-AGE antibody positive staining

\% RAGE mRNA: the percentage of cells positive for RAGE mRNA

$*$ p $<0.01$ vs DN

strong: 3 , very strong: 4$)$.

\section{in situ hybridization}

The probe for RAGE corresponded to sequences No. 784812 of human RAGE cDNA. One hundred picomoles of the oligonucleotide probe were labeled using a digoxigenin (DIG) oligonucleotide tailing kit according to the standard protocol (Roche, Penzberg, Germany). Free DIG was removed by ethanol precipitation and dissolved in diethylpyrocarbonate-treated water. In situ hybridization was performed according to the modified technique developed in our laboratory $(22,23)$. Briefly, fresh kidney biopsy tissues were embedded in OCT compound and stored at $-70^{\circ} \mathrm{C}$ until use. Specimens were cut into $4 \mu \mathrm{m}$ thick sections, fixed in $4 \%$ paraformaldehyde, and then deproteinized by $\mathrm{HCl}$ and digested with proteinase K (Sigma, St. Louis, MO). Specimens were prehybridized in a prehybridization buffer, drained and hybridized overnight with DIG-labeled oligonucleotide probe in the prehybridization buffer. After hybridization, the DIG-labeled probe was visualized by immunohistochemical staining using a mouse monoclonal anti-DIG antibody (Roche), HRP-conjugated rabbit anti-mouse antibody

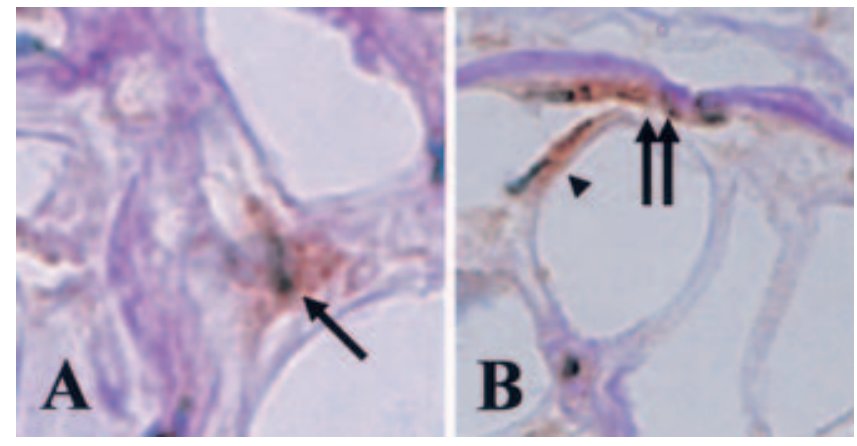

Figure 2. In situ hybridization of RAGE in glomeruli of diabetic nephropathy. In situ hybridization followed by PAS staining revealed that RAGE mRNAs were localized in glomerular resident cells, mainly glomerular mesangial cells (A: arrow), epithelial cells (B: arrowhead) and epithelial cells of Bowman's capsule (B: double arrow). (Original magnification $\times 200$ )

(Dako, Glostrup, Denmark), and HRP-conjugated swine anti-rabbit antibody (Dako). Color was developed with diaminobenzidine tetrahydrochloride in $0.05 \mathrm{M}$ Tris- $\mathrm{HCl}, \mathrm{pH}$ 7.6, and $0.03 \% \mathrm{H}_{2} \mathrm{O}_{2}$. Sections were counterstained briefly with hematoxylin, and then rinsed, dehydrated, cleared in xylene and mounted.

To evaluate the specificity of the signals, two types of control experiments were carried out as described previously $(22,23)$. First, pre-treatment with RNase was performed after proteinase $\mathrm{K}$ digestion, and thereafter sections were prehybridized and hybridized. Second, a competitive study was performed by adding 100-fold excess amount of homologous or unrelated, unlabeled oligonucleotides to the hybridization buffer together with the antisense probe.

To quantify the expression of RAGE mRNA, all nuclei as well as nuclei surrounded by RAGE-positive cytoplasm in at least 10 randomly selected cross-sections of nonsclerotic glomeruli were counted in each specimen. Enumeration of these nuclei was performed by three investigators who were 


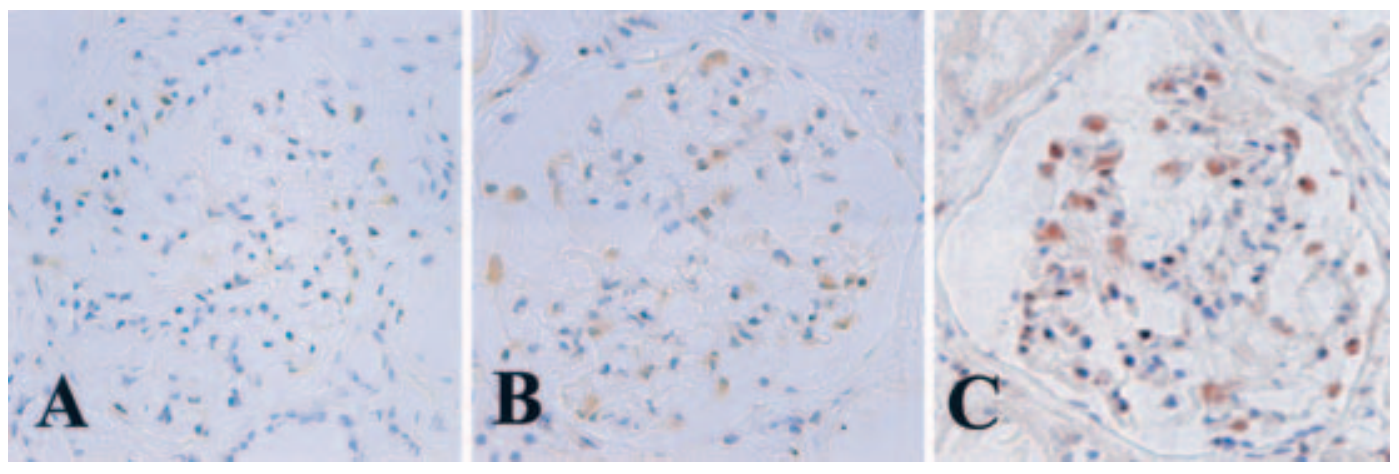

Figure 3. Detection of RAGE mRNA in tissues of normal controls (A) and diabetic nephropathy (B, with mild mesangial expansion and $\mathrm{C}$, with moderate mesangial expansion). (Original magnification $\times 100$ )

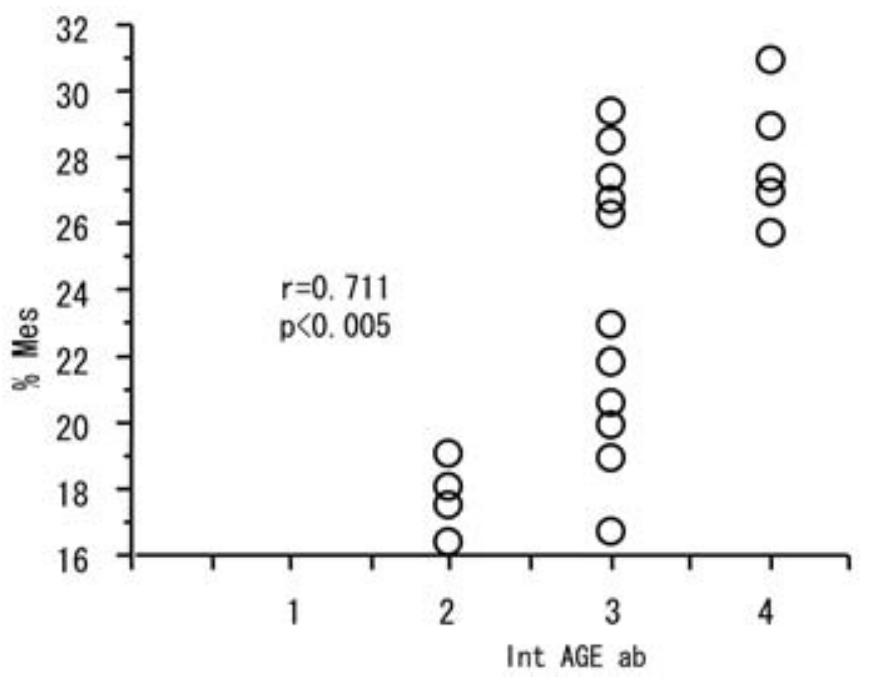

Figure 4. Correlation between percentage of mesangial area with respect to total glomerular area (\%Mes) and intensity of staining for anti-AGE antibody (Int AGE ab) in diabetic nephropathy. \% Mes correlated significantly with Int AGE ab.

blinded to the severity of DN. Results were expressed as the percentage of RAGE mRNA-positive cells among total glomerular cells.

\section{Statistical analysis}

The Mann-Whitney U test, regression analysis and Spearman rank correlation test were used for statistical comparison. A P value $<0.05$ denoted a statistically significant difference between groups.

\section{Results}

Immunohistochemical staining demonstrated the protein expression level of AGE in renal tissue of DN and control. The anti-AGE antibody was detected in the expanded mesangial matrix in DN tissues. The intensity of immunostaining appeared to increase with the degree of mesangial expansion in DN (Fig. 1 and Table 2). However, it was not de-

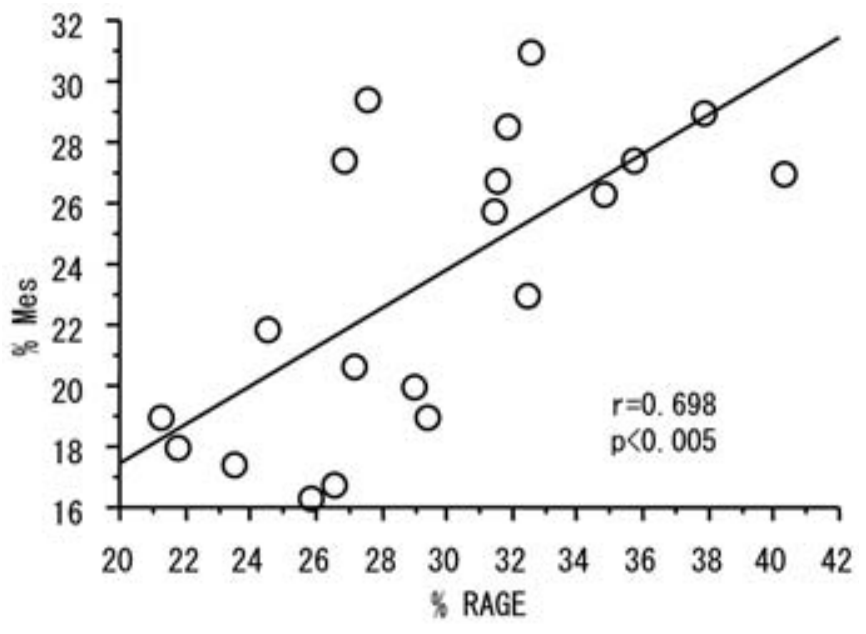

Figure 5. Correlation between percentage of mesangial area with respect to total glomerular area (\% Mes) and percentage of RAGE mRNA-positive cells among total glomerular cells (\% RAGE) in diabetic nephropathy. \% Mes correlated significantly with \% RAGE.

tected in control samples (Fig. 1). The intensity of staining for anti-AGE antibody was stronger in DN than in control samples (Table 2). Immunohistochemical staining using normal mouse IgG in lieu of this antibody was barely detectable in renal tissues from patients with DN (not shown).

Our in situ hybridization method also demonstrated cells positive for RAGE mRNA in renal tissue of DN and control. As shown in Fig. 2, in situ hybridization followed by PAS staining clearly identified individual cells positive for RAGE mRNA in renal tissue of DN and control. These cells included glomerular mesangial and epithelial cells and cells of Bowman's capsule (Fig. 2). Glomerular expression was noted to increase with the progression of mesangial expansion in DN. The percentage of cells positive for RAGE mRNA was significantly higher in DN than in control samples (Fig. 3, Table 2).

To verify the specificity of the signal obtained in in situ hybridization, two control studies were performed. Pre- 


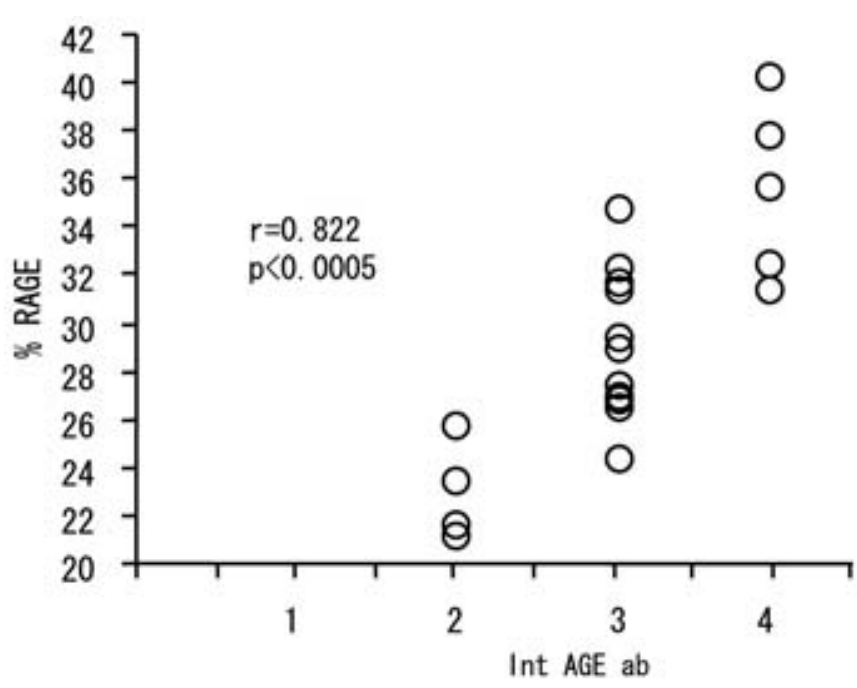

Figure 6. Correlation between percentage of RAGE mRNA-positive cells among total glomerular cells (\% RAGE) and intensity of staining for anti-AGE antibody (Int AGE ab) in diabetic nephropathy. \%RAGE correlated significantly with Int AGE ab.

treatment of tissue with RNase prior to hybridization eliminated most of the signal obtained with the RAGE probes (data not shown). The signal of each mRNA disappeared when a large amount of unlabeled homologous oligonucleotide was added. In contrast, the addition of non-homologous oligonucleotide did not block the signal (data not shown).

We also examined the relationships among \% Mes, the intensity of staining for anti-AGE antibody and the percentage of cells positive for RAGE mRNA. \%Mes correlated significantly with both the intensity of anti-AGE antibody-positive staining, and the percentage of cells positive for RAGE mRNA (Figs. 4, 5). The intensity of anti-AGE antibodypositive staining correlated significantly with the percentage of cells positive for RAGE mRNA (Fig. 6).

\section{Discussion}

AGE comprise a variety of molecular structures, such as CML (24), pentosidine (25), and pyrraline (26), characterized by different formation mechanisms. We recently demonstrated that anti CML and anti pentosidine antibodies were stained in the expanded mesangial matrix and nodular lesions of DN (8). The staining pattern for these antibodies was similar to the present study. Moreover, the intensity of positive anti-CML and pentosidine antibody staining appeared to increase with the degree of mesangial expansion in DN. The results of our present study also demonstrated positive staining for monoclonal anti-AGE antibody in the mesangium of DN, but this was not detected in control samples. Furthermore, the intensity of positive anti-AGE antibody staining correlated significantly with \%Mes. These data suggest that deposition and/or formation of AGE in the mesangium is associated with the progression of DN. In this study, we used just a semi-quantitative method employing the score of 0-4 to evaluate the intensity of staining for antiAGE antibody. A real quantitative method such as the direct evaluation of intensity of staining for antibodies is necessary to precisely quantify.

AGE mediate their effects through two different pathways via a receptor-independent AGE cross-link formation pathway and through a receptor-dependent pathway where AGE bind to specific cell surface-associated receptors, such as the macrophage scavenger receptor (MSR) type II, OST-48, 80K-H, galectin-3, CD 36 and $\operatorname{RAGE}(13,14,27-30)$. Among these receptors, RAGE is the best characterized and most implicated with the role of AGE. For that reason, we used the RAGE probe in this study. To evaluate the relationship between AGE and RAGE in tissues from DN, we also examined the expression of RAGE mRNA. Renal cells positive for RAGE mRNA were identified at the cellular level in DN and control. In order to distinguish individual cells positive for RAGE mRNA in the glomeruli, we performed PAS staining after in situ hybridization and then defined the topographical relationship of positively stained cells relative to GBM and mesangial matrix. The signal for RAGE mRNA was identified among glomerular resident cells, mainly glomerular mesangial and epithelial cells and cells of Bowman's capsule. Tanji et al (31) reported that RAGE protein expression was restricted to podocytes in normal control human glomeruli and in glomeruli of patients with DN. In another study, Tsuji et al (32) also demonstrated that both RAGE mRNA and RAGE protein were produced by mouse mesangial cells. Taken together, these results suggest that RAGE may be synthesized primarily by glomerular epithelial and mesangial cells. It is very interesting to investigate the expression of anti RAGE antibody. We had tried to do immunohistochemical staining using several anti RAGE antibodies. However, these antibodies did not react with RAGE on the tissues from control and DN. Further studies are warranted.

Our quantitative analysis of in situ hybridization studies demonstrated that the percentage of cells positive for RAGE mRNA was significantly greater in DN than in control samples. Tanji et al (31) reported that diffuse low-level expression of anti-RAGE antibody was restricted to the podocytes of normal control samples. On the other hand, there was marked upregulation of RAGE expression in tissues from DN. They also demonstrated RAGE mRNA expression in glomeruli from DN using RT-PCR. However, no expression was observed in glomeruli from normal control samples. Together, these data suggest that RAGE is upregulated in glomeruli in DN.

Recent studies reported that AGE-RAGE interaction is associated with the pathogenesis of DN. Yamamoto et al (33) established transgenic mice that overexpressed human RAGE in vascular cells and crossbred them with another transgenic line that develops insulin-dependent diabetes shortly after birth. The resultant double transgenic mice exhibited increased hemoglobin A1c and serum AGE levels, as did the diabetic controls. However, the double transgenic 
mice exhibited enlargement of the kidney, glomerular hypertrophy, increased albuminuria, mesangial expansion, advanced glomerulosclerosis, and increased serum creatinine compared with diabetic littermates lacking the RAGE transgene (33). Furthermore, treatment with neutralizing RAGE antibody or soluble RAGE improved renal function and renal pathological changes in $\mathrm{db} / \mathrm{db}$ mice $(34,35)$. To date, no studies have quantified RAGE mRNA and correlated the percentage of RAGE-expressing cells to the severity of diabetic glomerulopathy. The present study is the first to show that the percentage of cells positive for RAGE mRNA corre- lated significantly with not only \%Mes, but also with the intensity of anti-AGE antibody staining in glomeruli from DN. Moreover, it has been reported that AGE can activate the RAGE gene through NF- $\mathrm{KB}$ (36). Taken together, these data suggest that AGE accumulation may involve upregulation and activation of RAGE. The AGE-RAGE interaction apparently plays an important role in the pathogenesis of $\mathrm{DN}$. However, it is not clear whether the RAGE-independent AGE cross-link formation pathway or the RAGE-dependent pathway is more closely associated with development of DN. Further studies are required to address this issue.

\section{References}

1. Steffes MW, Osterby R, Chavers B, Mauer SM. Mesangial expansion as a central mechanism for loss of kidney function in diabetic patients. Diabetes 38: 1077-1081, 1989.

2. Suzuki D. Measurement of the extracellular matrix in glomeruli from patients with diabetic nephropathy using an automatic image analyzer. Nippon Jinzo Gakkai shi (Jpn J Nephrol) 36: 1209-1215, 1994.

3. Suzuki D, Takano H, Toyoda M, et al. Evaluation of renal biopsy samples of patients with diabetic nephropathy. Intern Med 40: 1077-1084, 2001

4. Mauer SM, Steffes MW, Brown DM. The kidney in diabetes. Am J Med 70: 603-612, 1981.

5. Fioretto P, Steffes MW, Sutherland DER, Goetz FC, Mauer M. Reversal of lesions of diabetic nephropathy after pancreas transplantation. N Engl J Med 339: 69-75, 1998.

6. Brownlee M, Cerami A, Vlassara H. Advanced glycosylation end products in tissue and the biochemical basis of diabetic complications. N Engl J Med 318: 1315-1321, 1988.

7. Suzuki D, Yagame M, Naka R, et al. Immunohistochemical staining of renal biopsy samples in patients with diabetic nephropathy in non-insulin dependent diabetes mellitus using monoclonal antibody to advanced glycation end products. Nephrology 1: 199-205, 1995.

8. Suzuki D, Miyata T, Saotome N, et al. Immunohistochemical evidence for an increased oxidative stress and carbonyl modification of proteins in diabetic glomerular lesions. J Am Soc Nephrol 10: 822-832, 1999.

9. Sell DR, Lapolla A, Odetti P, Forgarty J, Monnier VM. Pentosidine formation in skin correlates with severity of complication in individuals with long standing IDDM. Diabetes 41: 1286-1292, 1992.

10. McCance DR, Dyer DG, Dunn JA, Bailie KE, Thrope SR, Baynes JW, Lyons TJ. Maillard reaction products and their relation to complications in insulin-dependent diabetes mellitus. J Clin Invest 91: 2470-2478, 1993

11. Beisswenger PJ, Moore LL, Brink-Johnsen T. Increased collagenlinked pentosidine levels and advanced glycosylation end products in early diabetic nephropathy. J Clin Invest 92: 212-217, 1993.

12. Horie K, Miyata T, Maeda K, et al. Immunohistochemical colocalization of glycoxidation products and lipid peroxidation products in diabetic renal glomerular lesions. J Clin Invest 100: 29953004, 1997.

13. Schmidt AM, Vianna M, Gerlach $M$, et al. Isolation and characterization of two binding proteins for advanced glycation end products from bovine lung which are present on the endothelial cell surface. J Biol Chem 267: 14987-14997, 1992.

14. Neeper M, Schmidt AM, Brett J, et al. Cloning and expression of a cell surface receptor for advanced glycosylation end products of proteins. J Biol Chem 267: 14998-15004, 1992.

15. Skolnik EY, Yang Z, Makita Z, Radoff S, Kirstein M, Vlassara H.
Human and rat mesangial cell receptors for glucose-modified proteins: potential role in kidney tissue remodeling and diabetic nephropathy. J Exp Med 174: 931-939, 1991.

16. Doi T, Vlassara H, Kirstein M, Yamada Y, Striker GE, Striker LJ. Receptor-specific increase in extracellular matrix production in mouse mesangial cells by advanced glycosylation end products is mediated via platelet-derived growth factor. Proc Natl Acad Sci USA 89: 2873-2877, 1992.

17. Brett J, Schmidt AM, Yan SD, et al. Survey of the distribution of a newly characterized receptor for advanced glycation end products in tissues. Am J Pathol 143: 1699-1712, 1993.

18. Yamamoto Y, Kato I, Doi T, et al. Development and prevention of advanced diabetic nephropathy in RAGE-overexpressing mice. J Clin Invest 108: 261-268, 2001.

19. Wendt TM, Tanji N, Guo J, et al. RAGE drives the development of glomerulosclerosis and implicates podocyte activation in the pathogenesis of diabetic nephropathy. Am J Pathol 162: 11231137, 2003.

20. Nomoto Y, Tomino Y, Endoh M, et al. Modified open renal biopsy; Results in 934 patients. Nephron 45: 224-228, 1987.

21. Ikeda K, Higashi T, Sano H, et al. $\mathrm{N}^{\varepsilon}$-(carboxymethyl)lysine protein adduct is a major immunological epitope in proteins modified with advanced glycation end products of the Maillard reaction. Biochemistry 35: 8075-8083, 1996.

22. Suzuki D, Miyazaki M, Naka R, et al. in situ hybridization of interleukin 6 in diabetic nephropathy. Diabetes 44: 1233-1238, 1995.

23. Suzuki D, Miyazaki M, Jinde K, et al. In situ hybridization studies of matrix metalloproteinase-3, tissue inhibitor of metalloprotein-1 and type IV collagen in diabetic nephropathy. Kidney Int 52: 111-119, 1997.

24. Ahmed MU, Thorpe SR, Baynes JW. Identification of $\mathrm{N}^{\varepsilon}$ carboxymethyllysine as a degradation product of fructoselysine in glycated protein. J Biol Chem 261: 4889-4894, 1986.

25. Sell DR, Monnier VM. Structure elucidation of a senescence cross-link from human extracellular matrix. J Biol Chem 264: 21597-21602, 1989.

26. Njoroge FG, Sayre LM, Monnier VM. Detection of glucosederived pyrrole compounds during Maillard reaction under physiological conditions. Carbohydr Res 167: 211-220, 1987.

27. el Khoury J, Thomas CA, Loike JD, Hickman SE, Cao L, Silverstein SC. Macrophages adhere to glucose-modified basement membrane collagen IV via their scavenger receptors. J Biol Chem 269: 10197-10200, 1994.

28. Vlassara H, Li YM, Imani F, et al. Identification of galectin-3 as a high-affinity binding protein for advanced glycation end products (AGE): a new member of the AGE-receptor complex. Mol Med 1: 634-646, 1995.

29. Li YM, Mitsuhashi T, Wojciechowicz D, et al. Advanced glycation end products induce glomerular sclerosis and albuminuria in nor- 
mal rats. Proc Natl Acad Sci 93: 11047-11052, 1996.

30. Ohgami N, Nagai R, Ikemoto M, et al. CD36 serves as a receptor for advanced glycation end products (AGE). J Diabetes Complications 16: 56-59, 2002.

31. Tanji N, Markowitz GS, Fu C, et al. The expression of advanced glycation end products and their cellular receptor RAGE in diabetic nephropathy and non-diabetic renal disease. J Am Soc Nephrol 11: 1656-1666, 2000.

32. Tsuji $\mathrm{H}$, Iehara N, Masegi $\mathrm{T}$, et al. Ribozyme targeting of receptor for advanced glycation end products in mouse mesangial cells. Biochem Biophys Res Commun 245: 583-588, 1998.

33. Yamamoto Y, Kato I, Doi T, et al. Development and prevention of advanced diabetic nephropathy in RAGE-overexpressing mice. J Clin Invest 108: 261-268, 2001.
34. Flyvbjerg A, Denner L, Schrijvers BF, et al. Long-term renal effects of a neutralizing RAGE antibody in obese type 2 diabetic mice. Diabetes 53: 166-172, 2004.

35. Wendt TM, Tanji N, Guo J, et al. RAGE drives the development of glomerulosclerosis and implicates podocyte activation in the pathogenesis of diabetic nephropathy. Am J Pathol 162: 11231137, 2003.

36. Tanaka N, Yonekura H, Yamagishi S, Fujimori H, Yamamoto Y, Yamamoto $\mathrm{H}$. The receptor for advanced glycation end products is induced by the glycation products themselves and tumor necrosis factor- $\alpha$ through nuclear factor- $\mathrm{\kappa B}$, and by $17 \beta$-estradiol through $\mathrm{Sp}-1$ in human vascular endothelial cells. J Biol Chem 275: 25781-25790, 2000.

(C) 2006 The Japanese Society of Internal Medicine

http://www.naika.or.jp/imindex.html 